\title{
Effect of molecular weight on the vibronic structure of a diketopyrrolopyrrole polymer
}

\author{
Sophia C. Hayes, ${ }^{1}$ Galatia Pieridou, ${ }^{1}$ Michelle Vezie, ${ }^{2}$ Sheridan Few, ${ }^{2}$ Hugo Bronstein, ${ }^{3,4}$ Iain \\ Meager $^{3}$, Iain McCulloch ${ }^{3,5}$, Jenny Nelson ${ }^{2}$
}

1. Department of Chemistry, University of Cyprus, P. O. Box 20537, 1678 Nicosia, Cyprus

2. Centre for Plastic Electronics and Department of Physics, Imperial College London, Prince Consort Road, London SW7 2AZ, United Kingdom.

3. Centre for Plastic Electronics and Department of Chemistry, Imperial College London, Exhibition Road, London SW7 2AZ, United Kingdom.

4. Department of Chemistry, University College London, 20 Gordon Street, LondonWC1H OAJ, United Kingdom.

5. SPERC, King Abdullah University of Science and Technology, Thuwal, 23955-6900, Saudi Arabia

\begin{abstract}
Resonance Raman Spectroscopy (RRS) is employed in this study to examine the influence of molecular weight on the optical response of a diketopyrrolopyrrole polymer (DPP-TT-T) in solution. The vibronic structure observed for the ground state absorption of this polymer is found to vary with molecular weight and solvent. Resonance Raman Intensity Analysis (RRIA) revealed that the absorption spectra can be described by at least two dipole-allowed transitions and the vibronic structure variation is due to differing contributions from linear and curved segments of the polymer.
\end{abstract}

Keywords: Resonance Raman spectroscopy, DPP-TT-T, conjugated polymer, vibronic structure, molecular weight.

\section{INTRODUCTION}

The vibronic structure observed in absorption spectra of conjugated polymers can hide important information regarding the electronic transition and it is tightly connected to the conformation of the polymer. Vibronic intensities are associated with the transition dipole moment and the change in the excited state structure relative to that of the ground state; however, vibronic intensities have been also related to polymer aggregation and order. ${ }^{1}$ In our recent work we have shown that there is an apparent maximum in the extinction coefficient of polymers with different chemical structure, self-organising tendency and optical gap $(\kappa \approx 1){ }^{2}$ From a large number of polymers studied, three polymers stood out (IDTBT, DPP3T, DPP-TT-T) that exemplified a remarkable absorption at low energies $(\kappa \approx 1.5-1.6)$, which has implications in their light-harvesting efficiency, leading to high photocurrent in solar cells. Our work focuses on DPPTT-T, a promising semicrystalline polymer with a planarised backbone, high hole mobility (\& ambipolar transport), promising performance in solar cells and ambient stability. It has been shown that the extinction coefficient for DPP-TT$\mathrm{T}$ varies with molecular weight as well as its optoelectronic properties. Interestingly, the vibronic structure exhibited by the absorption spectra is also affected by the molecular weight of this polymer. Here, we describe our efforts to elucidate the contribution of polymer conformation to the observed molecular weight dependence with the use of Resonance Raman Spectroscopy (RRS). RR band intensities are associated with structural changes upon electronic excitation and are thus directly related to the displacement between the ground and excited state potential energy surface minima along specific normal coordinates, which determine in turn the shape of absorption spectra. ${ }^{3}$ Quantification of the intensities of the RR bands and analysis in combination with the absorption spectrum (a method called resonance Raman intensity analysis) can provide valuable information for the excited state potential energy surface landscape in the Frank-Condon region, a difficult region to probe with other methods. ${ }^{3}$ We use this method to analyze the different vibronic structure

Physical Chemistry of Interfaces and Nanomaterials XV, edited by Artem A. Bakulin, Robert Lovrincic, Natalie Banerji, Proc. of SPIE Vol. 9923, 99230N · C 2016 SPIE · CCC code: 0277-786X/16/\$18 · doi: 10.1117/12.2238123 
exemplified by two different molecular weight fractions of DPP-TT-T, offering in addition an explanation for the high absorption exemplified by this polymer. ${ }^{2}$

\section{EXPERIMENTAL METHODS}

2.1. Materials. Dilute solutions $\left(\sim 5.5 \times 10^{-5} \mathrm{M}-6.5 \times 10^{-5} \mathrm{M}\right)$ of low and high molecular weight batches of DPP-TT-T were prepared in chloroform (CF) (HPLC, 99.9\%, Aldrich) and 1,2-dichlorobenzene (ODCB) (HPLC, 99.9\% Aldrich), which were used as received. The low molecular weight polymer had $\mathrm{Mw}=51 \mathrm{kDa}(\mathrm{Mn}=16 \mathrm{kDa})$ and the high molecular polymer $228 \mathrm{kDa}(\mathrm{Mn}=99 \mathrm{kDa})$. The exact concentration of each DPP-TT-T solution was obtained from the UV-Vis spectrum (Shimadzu), using the pseudo molar extinction coefficient of DPP-TT-T at high molecular weight (HMW) in CF at $823 \mathrm{~nm}\left(90 \times 10^{3} \mathrm{Lmol}^{-1} \mathrm{~cm}^{-1}\right)$ and at $811 \mathrm{~nm}$ in ODCB $\left(85 \times 10^{3} \mathrm{Lmol}^{-1} \mathrm{~cm}^{-1}\right)$. The pseudo molar extinction coefficient of low molecular weight DPP-TT-T (LMW) in CF was $70 \times 10^{3} \mathrm{Lmol}^{-1} \mathrm{~cm}^{-1}$ at $807 \mathrm{~nm}$ and $65 \times 10^{3}$ $\mathrm{Lmol}^{-1} \mathrm{~cm}^{-1}$ at $796 \mathrm{~nm}$ in ODCB.

2.2. Resonance Raman Studies. The absolute resonance Raman (RR) intensities of DPP-TT-T for the two molecular weights dissolved either in CF or ODCB were measured with excitation at 636.8, 682.9 and $737.8 \mathrm{~nm}$. The 682.9 and $636.8 \mathrm{~nm}$ excitation wavelengths employed in the RR experiments were produced via Raman shifting the second and third harmonics from a Q-switched Nd:YAG laser (PRO-230, $30 \mathrm{~Hz}$, Spectra Physics) at 532 and $355 \mathrm{~nm}$, respectively, in a homemade $1 \mathrm{~m}$ stainless-steel tube containing $\mathrm{H}_{2}$ gas at 1.5 bar. The $737.8 \mathrm{~nm}$ excitation wavelength was produced via Raman shifting the fundamental from a Q-switched Nd:YAG laser (Lab-190, $30 \mathrm{~Hz}$, Spectra Physics) at $1064 \mathrm{~nm}$, in a $1 \mathrm{~m}$ stainless-steel tube containing $\mathrm{H}_{2}$ gas at 40 bar (Light Age). The excitation light was focused into a spinning cell consisting of an EPR suprasil tube (diameter: $4 \mathrm{~mm}$ ) attached to a rheostat-controlled motor for choice of rotation speed. Use of the spinning cell prolonged the lifetime of the samples. Modest excitation energies $(\sim 15 \mu \mathrm{J}$ per pulse $)$ were employed to avoid decomposition of the sample. The Raman scattered light was collected in a backscattering geometry and delivered to a $0.75 \mathrm{~m}$ focal-length Czerny-Turner spectrograph, equipped with a 1200 -grooves $/ \mathrm{mm}$ holographic grating. The slit width was set to $100 \mu \mathrm{m}$ providing for $2 \mathrm{~cm}^{-1}$ spectral resolution at the wavelengths used in this work. The scattered light was detected by a thermoelectrically-cooled $1340 \times 400$ pixel, back-illuminated deep depletion CCD detector (PIXIS-XB:400 BR, Princeton Instruments). Each spectrum presented here is the accumulation of 12-24 $10 \mathrm{~min}$ spectra. Frequency calibration of the spectra was accomplished with the use of toluene. MATLAB and ORIGIN software were used for spectral treatment and analysis.

2.3. Determination of Absolute Resonance Raman Cross Sections. In the calculation of the absolute RR cross sections the $1002 \mathrm{~cm}^{-1}$ mode of toluene $(9.4 \mathrm{M})$ was used as an external standard. The intensities of the Raman bands were corrected for the spectral response of the instrument and were determined by peak integration using the trapezoidal rule. In congested spectral regions deconvolution of the bands was performed and the area of the Gaussian peaks was subsequently calculated. The intensities were also corrected for self-absorption according to Eq. $1:^{4}$

$$
\frac{I_{\text {scorrected }}}{I_{\text {rcorrected }}}=\frac{I_{S} c_{r}\left(\varepsilon_{S}+\varepsilon_{0}\right)}{I_{r} c_{S}\left(\varepsilon_{r}+\varepsilon_{0}\right)} \quad \text { (1) } \quad \sigma_{R(v(x))}=\frac{I_{v(x)} C_{i}\left(\frac{1+2 \rho}{1+\rho}\right)_{v(x)}}{I_{i} C_{v(x)}\left(\frac{1+2 \rho}{1+\rho}\right)_{i}} \sigma_{i}
$$

where $c$ is the concentration of the reference or sample in the material tested and $\varepsilon$ is the extinction coefficient of the sample at the reference or sample peak, or laser line wavelength (subscripts $r, s$ and 0 respectively). Absolute RR cross sections as a function of excitation wavelength were determined using Eq. 2 only for the high-frequency modes of C3 in $\mathrm{CF}$ and ODCB, as these were the most intense bands in the spectra. ${ }^{5}$ In Eq. $2, \sigma_{R}$ is the Raman scattering cross section of mode $v(\mathrm{x}), \rho$ is the depolarization ratio, $\mathrm{C}$ is the concentration, and $I_{v(x)}$ and $I_{i}$ are the experimentally determined intensities for the mode of interest and the external standard, respectively. The absolute Raman cross section and depolarization ratio for the $1002 \mathrm{~cm}^{-1}$ mode of toluene were previously measured ${ }^{6}$. The depolarization ratios for the modes of $\mathrm{C} 3$ were taken as $1 / 3$.

\section{RESULTS}

The absorption spectra for the two molecular weight fractions of DPP-TT-T in the two different solvents are presented in Figure 1. We can observe that the absorption cross section for the LMW polymer is significantly lower than for the 
HMW fraction. The vibronic intensities are also different with a reduced $0-0$ band intensity relative to the $0-1$ for the LMW polymer indicative of a different ground and excited state potential well alignment. Interestingly the solvent affects the vibronic intensities in a greater extent in the LMW case. We investigate the absorption cross section and vibronic intensity variations with the use of resonance Raman spectroscopy, exciting the polymers at various energies within the absorption band. The resonance Raman spectra of the polymers studied in ODCB with excitation at 683 and

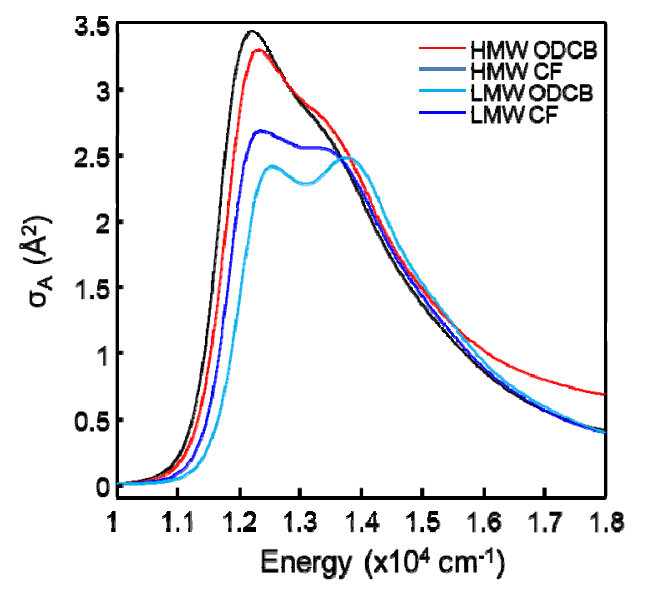

Figure 1. UV-vis absorption spectra of HMW and LMW DPP-TT-T in ODCB and CF. The absorbance is expressed in terms of absorption cross section.

$737 \mathrm{~nm}$ are presented in Figure 2. Very similar spectra were obtained in $\mathrm{CF}^{2}{ }^{2}$ We have recently reported detailed assignment of the Raman bands of DPP-TT-T via normal mode calculations for both linear ("trans") and curved ("cis") conformations of tetramers using Gaussian. ${ }^{2,7}$ As this copolymer contains diketopyrrolopyrrole (DPP) and thienothiophene (TT) and thiophene units, the RR spectra are expected to consist of vibrational bands that span the spectra of these units. Here, bands are observed at 1492 and $1477 \mathrm{~cm}^{-1}$ associated with the backbone of TT and
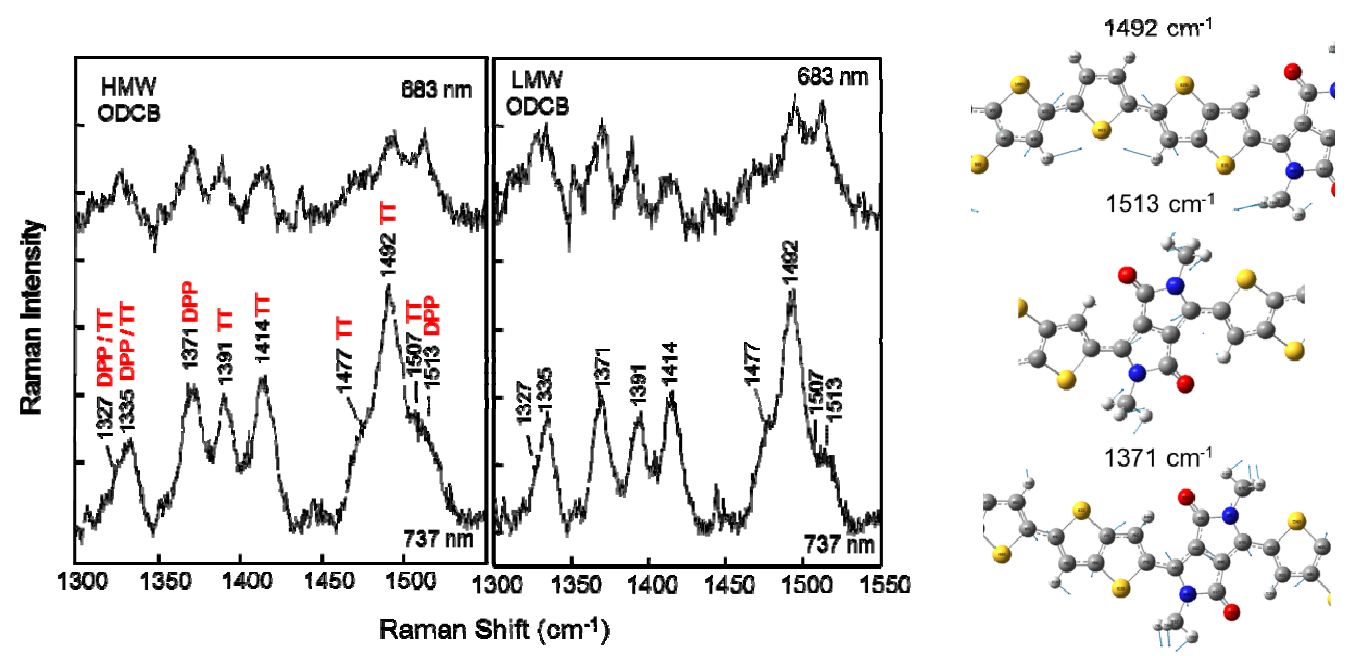

Figure 2. (left) Resonance Raman spectra of HMW and LMW DPP-TT-T polymers in ODCB with excitation at 683 and $737 \mathrm{~nm}$. Assignment of the bands is indicated according to the part of the monomer they correspond to. (right) Eigenvector description of key DPP-TT-T normal modes.

correspond primarily to $\mathrm{C}=\mathrm{C}$ stretching $(\mathrm{C}=\mathrm{C}$ on each side of $\mathrm{T}$ and $\mathrm{C}=\mathrm{C}$ stretching of one $\mathrm{C}=\mathrm{C}$ on each side of DPP, respectively). The bands observed at 1513 and 1371 are assigned to DPP modes, and specifically to $\mathrm{C}=\mathrm{C}$ s stretching (opposite sides opposite rings) and $\mathrm{C}-\mathrm{N}$ stretch and $\mathrm{C}=\mathrm{C}$ s stretch between rings, respectively. The peaks at 1414, 1391 
and $1335 \mathrm{~cm}^{-1}$ are assigned to both TT and DPP $\left(\mathrm{C}=\mathrm{C}\right.$ stretching in TT and umbrella mode of $\mathrm{CH}_{3}$ in DPP for the former two and $\mathrm{N}-\mathrm{CH}_{3}$ stretching in DPP and C-C inter-ring stretching between TT and DPP for the $1335 \mathrm{~cm}^{-1}$ mode).It is apparent that the relative intensities of the bands in the spectra of HMW and LMW C3 polymer at the same excitation wavelengths are very similar. However, the relative intensities of both polymers around $1500 \mathrm{~cm}^{-1}$ vary distinctively between excitation at $737 \mathrm{~nm}$ and $683 \mathrm{~nm}$, which indicates the involvement of different electronic states in the scattering, as the intensities of resonance Raman bands are associated with structural changes involving the particular coordinate upon excitation to a specific electronic excited state. ${ }^{8}$ The relative intensities of the RR spectra at $636 \mathrm{~nm}$, which is on the edge of the absorption band (not presented here) for the two molecular weights and two solvents examined, are very similar to the ones at $683 \mathrm{~nm}$ suggesting that the same state is accessed as with $683 \mathrm{~nm}$ excitation, but different than with $737 \mathrm{~nm}$ excitation. The most intense band in the spectra at $737 \mathrm{~nm}$ belongs to the $1492 \mathrm{~cm}^{-1}$ mode, associated with $\mathrm{C}=\mathrm{C}$ stretching in the thienothiophene unit (Figure 2). Large intensity in the RR spectra indicates a change in the $\mathrm{C}=\mathrm{C}$ bond lengths, in agreement with a transition from a benzenoid to a quinoidal structure in the excited state, consistent with calculated orbital diagrams of oligomers of DPP-TT-T (Figure 4). Excitation at $683 \mathrm{~nm}$, however, indicates that the 1513

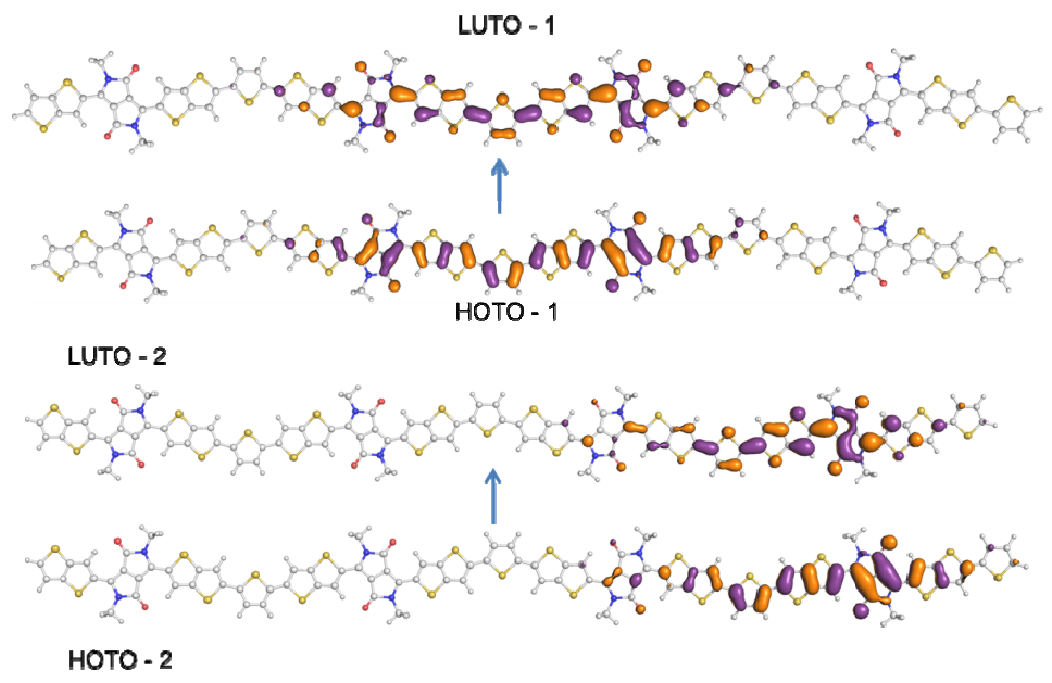

Figure 3. First (-1) and second (-2) natural orbital transitions for the 'trans' conformation of a DPP-TT-T tetramer. Calculated using the CAM-B3LYP-631g* basis set in Gaussian.

Table 1. Absolute resonance Raman Cross Sections $\left(\sigma_{R}\right)$ for the vibrational modes of DPP-TT-T at 737 and $683 \mathrm{~nm}$ included in RRIA analysis. $^{2}$

\begin{tabular}{|c|c|c|c|c|c|c|c|c|}
\hline $\begin{array}{c}\bar{v} \\
\left(\mathrm{~cm}^{-1}\right)\end{array}$ & $\begin{array}{c}\sigma_{\mathrm{R}}(737 \mathrm{~nm}) \\
\left(\mathrm{x} 10^{-9} \AA^{2}\right)\end{array}$ & $\begin{array}{c}\sigma_{\mathrm{R}}(683 \mathrm{~nm}) \\
\left(\mathrm{x} 10^{-9} \AA^{2}\right)\end{array}$ & $\begin{array}{c}\sigma_{\mathrm{R}}(737 \mathrm{~nm}) \\
\left(\mathrm{x} 10^{-9} \AA^{2}\right)\end{array}$ & $\begin{array}{c}\sigma_{\mathrm{R}}(683 \mathrm{~nm}) \\
\left(\mathrm{x} 10^{-9} \AA^{2}\right)\end{array}$ & $\begin{array}{c}\sigma_{\mathrm{R}}(737 \mathrm{~nm}) \\
\left(\mathrm{x} 10^{-9} \AA^{2}\right)\end{array}$ & $\begin{array}{c}\sigma_{\mathrm{R}}(683 \mathrm{~nm}) \\
\left(\mathrm{x} 10^{-9} \AA^{2}\right)\end{array}$ & $\begin{array}{c}\sigma_{\mathrm{R}}(737 \mathrm{~nm}) \\
\left(\mathrm{x} 10^{-9} \AA^{2}\right)\end{array}$ & $\begin{array}{c}\sigma_{\mathrm{R}}(683 \mathrm{~nm}) \\
\left(\mathrm{x} 10^{-9} \AA^{2}\right)\end{array}$ \\
\hline & \multicolumn{2}{|c|}{$\mathbf{L M W - O D C B}$} & \multicolumn{2}{|c|}{$\mathbf{L M W - C F}$} & \multicolumn{2}{|c|}{ HMW-ODCB} & \multicolumn{3}{|c|}{ HMW-CF } \\
\hline 1327 & $6.7 \pm 0.8$ & $3.9 \pm 0.9$ & $4.1 \pm 0.8$ & $3.1 \pm 1.2$ & $17.6 \pm 3.5$ & $4.9 \pm 0.3$ & $12.0 \pm 8.9$ & $4.0 \pm 1.8$ \\
\hline 1335 & $18.0 \pm 3.3$ & $4.6 \pm 1.2$ & $15.9 \pm 3.6$ & $4.0 \pm 1.5$ & $21.5 \pm 3.2$ & $3.2 \pm 0.9$ & $17.3 \pm 6.9$ & $3.9 \pm 1.8$ \\
\hline 1371 & $28.9 \pm 4.8$ & $10.0 \pm 3.4$ & $27.7 \pm 7.8$ & $8.5 \pm 2.2$ & $62.1 \pm 15.3$ & $13.0 \pm 3.0$ & $71.4 \pm 10.1$ & $15.2 \pm 4.6$ \\
\hline 1391 & $25.2 \pm 4.6$ & $7.6 \pm 2.7$ & $28.1 \pm 6.6$ & $3.8 \pm 0.6$ & $52.3 \pm 8.6$ & $11.1 \pm 7.6$ & $66.1 \pm 6.7$ & $11.7 \pm 1.6$ \\
\hline 1414 & $33.6 \pm 5.9$ & $10.2 \pm 2.5$ & $34.5 \pm 7.0$ & $4.5 \pm 1.4$ & $71.2 \pm 11.2$ & $10.3 \pm 3.0$ & $88.9 \pm 19.6$ & $9.3 \pm 3.3$ \\
\hline 1477 & $23.7 \pm 5.9$ & $4.0 \pm 1.2$ & $21.8 \pm 5.6$ & $9.2 \pm 1.1$ & $52.6 \pm 11.0$ & $3.7 \pm 2.5$ & $65.8 \pm 12.8$ & $21.0 \pm 8.7$ \\
\hline 1492 & $49.4 \pm 9.1$ & $10.8 \pm 3.2$ & $40.2 \pm 11.2$ & $5.7 \pm 0.7$ & $81.6 \pm 19.7$ & $18.4 \pm 8.3$ & $84.2 \pm 14.7$ & $12.4 \pm 3.3$ \\
\hline 1507 & $6.1 \pm 2.7$ & - & $9.5 \pm 2.4$ & - & $18.9 \pm 0.8$ & - & $22.9 \pm 12.4$ & - \\
\hline 1513 & $18.5 \pm 6.3$ & $20.2 \pm 6.5$ & $15.94 \pm 4.2$ & $14.2 \pm 0.9$ & $42.6 \pm 15.7$ & $23.0 \pm 10.9$ & $65.9 \pm 5.9$ & $28.6 \pm 5.1$ \\
\hline
\end{tabular}


$\mathrm{cm}^{-1}$ band associated with the DPP unit is gaining significant intensity relative to the $1492 \mathrm{~cm}^{-1}$ band, with absolute RR cross sections almost twice as large (Table 1). Similar change in relative intensities is observed between the 1371 and $1414 \mathrm{~cm}^{-1}$ bands. The band associated with the DPP unit gains intensity at $683 \mathrm{~nm}$ with respect to the band associated with the TT. This suggests that the higher energy state accessed sustains different electron localization in the DPP unit. Observation of the orbital diagrams for the lower energy transition reveals a nodal point at the $\mathrm{C}=\mathrm{C}$ bond between the pyrrole rings in the DPP unit while large electron density resides on this bond in the case of the higher energy transition (Figure 3).

We use the information obtained from the resonance Raman Resonance spectra above (frequencies and cross sections) in order to investigate the vibronic structure of the absorption spectra through Intensity Analysis (RRIA). This analysis entails the simultaneous modelling of the electronic absorption spectrum and the excitation-energy-dependent absolute RR cross sections (Raman excitation profile, REP), using expressions from the time-dependent formalism for absorption and Resonance Raman scattering. ${ }^{3}$ We note here that an initial fit to the absorption and RR cross sections for the two polymers in the two solvents was attempted using a single dipole-allowed transition in the model. While the fit to the absorption was quite satisfactory, the RR cross sections were overestimated. The largest error was found in the case of the $1492 \mathrm{~cm}^{-1}$, the most intense band in the RR spectra, where the calculated cross section is off by about a factor of 9 , while a factor of 2 off the experimental values is found in the case of the other modes. Considering the change in relative intensities observed at the two different excitation wavelengths, we have modified the equations for the absorption and Raman cross sections to reflect the involvement of two electronic transitions:

$$
\begin{aligned}
& \sigma_{A}\left(E_{L}\right)=\frac{4 \pi e^{2} E_{L}}{6 \hbar^{2} c n} \int_{-\infty}^{\infty} d E_{00} H\left(E_{00}\right) \times\left[\int_{-\infty}^{\infty} M_{1}^{2}\left\langle i \mid i_{1}(t)\right\rangle \exp \left[\frac{i\left(E_{L}+\epsilon_{i}\right) t}{\hbar}\right] D_{1}(t) d t+\int_{-\infty}^{\infty} M_{2}^{2}\left\langle i \mid i_{2}(t)\right\rangle \exp \left[\frac{i\left(E_{L}+\epsilon_{i}\right) t}{\hbar}\right] D_{2}(t) d t\right] \\
& \sigma_{i \rightarrow f}\left(E_{L}\right)=\frac{8 \pi e^{4} E_{S} E_{L}}{9 \hbar^{6} c^{4}} \int \partial E_{00} H\left(E_{00}\right)\left|M_{1}^{2} \int_{0}^{\infty}\left\langle f \mid i_{1}(t)\right\rangle \exp \left(\frac{i\left(E_{L}+\epsilon_{i}\right) t}{\hbar}\right) D_{1}(t) d t+M_{2}^{2} \int_{0}^{\infty}\left\langle f \mid i_{2}(t)\right\rangle \exp \left(\frac{i\left(E_{L}+\epsilon_{i}\right) t}{\hbar}\right) D_{2}(t) d t\right|^{2}
\end{aligned}
$$

In the above equations, $E_{S}$ and $E_{L}$ are the scattered and incident photon energies, respectively (in $\left.\mathrm{cm}^{-1}\right), c$ is the speed of light, the magnitude of the transition dipole moment for the electronic transition is given by $\mathrm{M}$ (in $\AA$ ), $\epsilon_{i}$ is the energy of the initial vibrational state and $n_{r}$ is the solvent index of refraction. $\Gamma$ is the homogeneous line width of the excited state (in $\mathrm{cm}^{-1}$ ), and is given by the damping function $\mathrm{D}(\mathrm{t})$. In this analysis the homogeneous broadening was taken to be Gaussian $\left(D(t)=e^{-\frac{\Gamma^{2} t^{2}}{\hbar^{2}}}\right)$. $\mathrm{E}_{00}$ is the energy difference between the $v=0$ vibrational state in the ground and excited electronic state and $\mathrm{H}\left(\mathrm{E}_{00}\right)$ is the contribution of inhomogeneous broadening, which corresponds, for example, to the presence of different solvent sites that are static on the time scale of Raman scattering, thus altering $\mathrm{E}_{00}$. The expression $\left\langle f \mid i_{n}(t)\right\rangle$ represents the time-dependent overlap of the final state in the scattering process with the initial state propagating under the influence of the excited state Hamiltonian in state $n$. In the expression for the absorption cross section $\left\langle i \mid i_{n}(t)\right\rangle$ is the time-dependent overlap of the initial ground vibrational state with the same state propagating on the excited state $n$ potential energy surface. ${ }^{3}$ In the case where both ground and excited state normal coordinates are identical and harmonic, the multidimensional overlaps $\langle i \mid i(t)\rangle$ and $\langle f \mid i(t)\rangle$ can be described by one dimensional overlaps in each normal coordinate. Furthermore, in fundamental Raman scattering, where the final state differs from the initial state only in one coordinate, the multidimensional overlaps $\langle f \mid i(t)\rangle$ can be decomposed into $\langle f \mid i(t)\rangle$ for the Raman active mode and $\langle i \mid i(t)\rangle$ that refers to all other modes. ${ }^{3}$ Explicit expressions have been derived ${ }^{3}$ for the onedimensional overlaps, where the dependence on the displacement, $\Delta\left(\mathrm{s}=\Delta^{2} / 2\right)$, between the ground and excited state potential energy surface minima along each coordinate, is apparent:

$$
\begin{aligned}
& \langle i \mid i(t)\rangle=\exp \left[-s\left(1-e^{-i \omega t}\right)-\frac{i \omega t}{2}-\frac{i E_{0} t}{\hbar}\right] \\
& \langle f \mid i(t)\rangle= \pm s^{1 / 2}\left(e^{-i \omega t}-1\right)\langle i \mid i(t)\rangle
\end{aligned}
$$

The time-dependent overlaps here were calculated using the methodology of Yan and Mukamel ${ }^{9}$, using a time step of 0.1 fs for a total of 5000 points. It is important to note that in the case where two electronic transitions are involved, the transition polarizabilities for each vibrational mode associated with each transition are added and squared to give the Raman cross section and can then be expressed as follows: ${ }^{10}$

$$
\sigma_{R} \propto\left|a_{1}+a_{2}\right|^{2}=\left|a_{1}\right|^{2}+\left|a_{2}\right|^{2}+a_{1} \cdot a_{2} *+a_{1} * a_{2}
$$


One can observe that in this case the interference terms in Eq. 7, $\alpha_{1} \alpha_{2} *$ and $\alpha_{1} * \alpha_{2}$, depend on the product of the displacements $\boldsymbol{\Delta}_{\mathbf{1}} \boldsymbol{\Delta}_{\mathbf{2}}$ and the signs of these interference terms depend on the relative signs of the two displacements. The interference effects are expected to be greatest when the two states have comparable transition dipole moments and where there is a large region of overlap between the two transitions. ${ }^{10}$ However, the sign of $\Delta$ does not affect the absorption cross section because $s$ is a function of $\Delta^{2}$.

Considering two dipole-allowed transitions in the model produces the fits presented in Figure 4, where both the absorption spectra of the two DPP-TT-T polymers in the two solvents and the REPS for the most intense vibrational modes are fit simultaneously well. Here we only show the Raman excitation profile for the $1492 \mathrm{~cm}^{-1}$ mode, which is primarily coupled to the electronic transition (largest $\Delta$ ). Key to the best fit of the data was the interference between the two transitions, where the opposite sign of the displacements helped reduce the calculated RR cross sections. All the parameters used in the model are reported in Table 2. Therefore the high energy shoulder observed experimentally in the absorption spectrum can be assigned to the sum of two contributions, the second vibronic peak of the first electronic transition and the first vibronic peak of the second electronic transition. The position of the second electronic transition was very sensitive and was optimized to $160 \mathrm{meV}$ above the first. Calculations of the vertical excitation energies for tetramers of DPP-TT-T indicated a second transition at around $170 \mathrm{meV}$ above the first. ${ }^{2}$ However, this second calculated transition is dark for linear oligomers but moderately bright in curved (all-cis) oligomers with transition dipole moment $\boldsymbol{\mu}_{02}$ oriented perpendicular to the chain. The necessity for a close by transition in the model strongly suggests the presence of a mixture of conformations in solution, as theoretically the second transition gains oscillator strength only in the cis conformation. Interestingly, the transition dipole moment for the lowest energy transition is found higher in the HMW case, with the ratio $\mathrm{M}_{1} / \mathrm{M}_{2}=1.57$ vs. 1.45 for the LMW DPP-TT-T. This is indicative for a greater fraction of linear conformers in the HMW polymer. In our recent work we have correlated the oscillator strength to chain linearity through calculation of the persistence length for a variety of polymers. ${ }^{2}$ Therefore, we can conclude that the higher overall oscillator strength observed in the HMW DPP-TT-T is due to this greater proportion of linear conformers. We suggest that the higher tendency of chains to adopt a linear conformation in the HMW case is due to the increased strength of chain-chain interactions (which will be maximized for linear chains) over the chain-solvent interactions, consistent with the lower solubility of the longer chains. The hypothesis that chain-chain interactions are more important for higher MW is consistent with the stronger effect of solvent on the absorption spectrum for the LMW than the HMW polymer (Fig. 1).
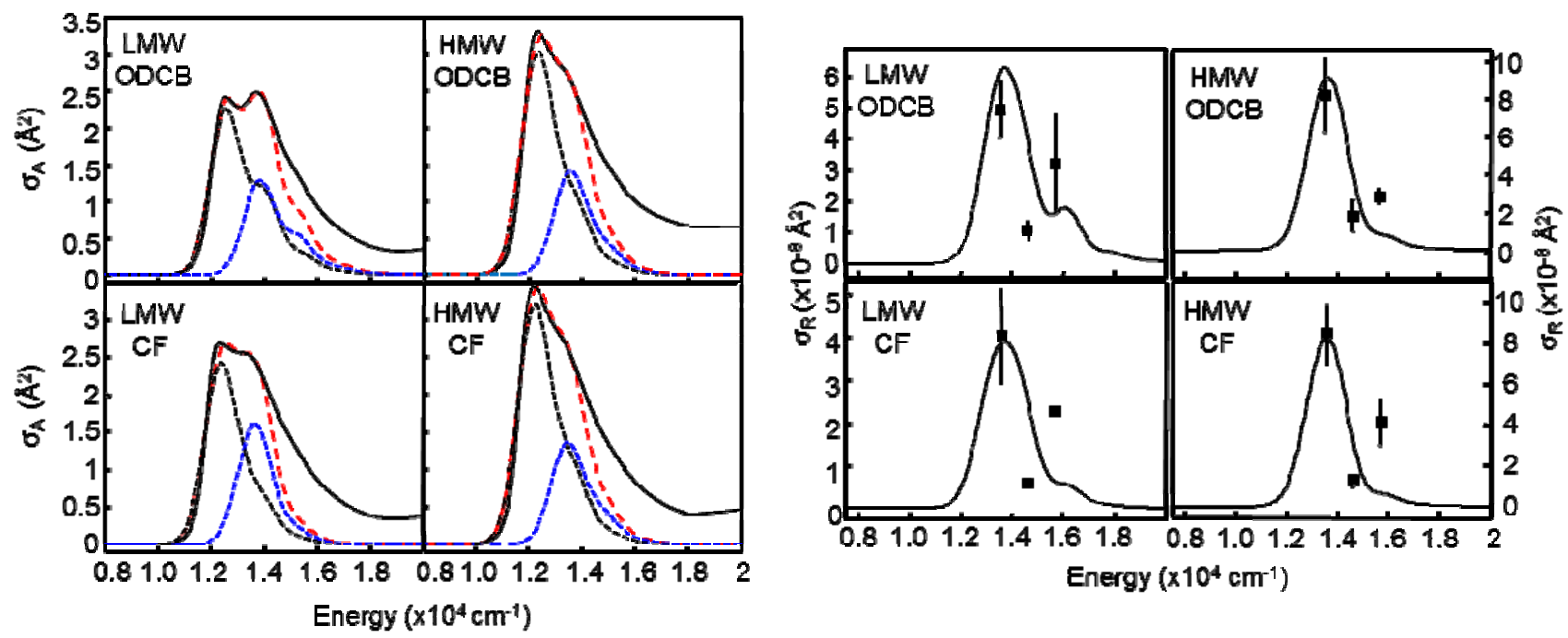

Figure 4. (Left) Experimental (solid black line) and calculated (dashed red line) absorption cross sections of LMW and HMW DPPTT-T polymers in ODCB (upper plots) and CF (lower plots). The dashed black and blue lines represent the absorption spectra for the two transitions that contribute to the overall absorption band. (Right) Experimental (points) and calculated (solid line) Raman excitation profiles for the $1492 \mathrm{~cm}^{-1}$ mode of LMW and HMW DPP-TT-T polymer in ODCB (upper plots) and CF (lower plots) using two dipole-allowed transitions in the RRIA. 
Table 2. Parameters employed in the RRIA of the LMW and HMW DPP-TT-T polymers in ODCB in the case of two dipole-allowed transitions. ${ }^{2}$

\begin{tabular}{|c|c|c|c|c|c|c|c|c|c|c|c|c|}
\hline Transition & $\begin{array}{c}\omega_{g} \\
\left(\mathrm{~cm}^{-1}\right)^{\mathrm{a}}\end{array}$ & $\begin{array}{c}\omega_{e} \\
\left(\mathrm{~cm}^{-1}\right)^{\mathrm{b}}\end{array}$ & $\Delta_{\mathrm{k}}^{\mathrm{c}}$ & $\begin{array}{c}\omega_{g} \\
\left(\mathrm{~cm}^{-1}\right)^{\mathrm{a}}\end{array}$ & $\begin{array}{c}\omega_{e} \\
\left(\mathrm{~cm}^{-1}\right)^{\mathrm{b}}\end{array}$ & $\Delta_{\mathrm{k}}^{\mathrm{c}}$ & $\begin{array}{c}\omega_{g} \\
\left(\mathrm{~cm}^{-1}\right)^{\mathrm{a}}\end{array}$ & $\begin{array}{c}\omega_{e} \\
\left(\mathrm{~cm}^{-1}\right)^{\mathrm{b}}\end{array}$ & $\Delta_{\mathrm{k}}^{\mathrm{c}}$ & $\begin{array}{c}\omega_{g} \\
\left(\mathrm{~cm}^{-1}\right)^{\mathrm{a}}\end{array}$ & $\begin{array}{c}\omega_{e} \\
\left(\mathrm{~cm}^{-1}\right)^{\mathrm{b}}\end{array}$ & $\Delta_{\mathrm{k}}^{\mathrm{c}}$ \\
\hline \multicolumn{4}{|c|}{ LMW ODCB-1 } & \multicolumn{3}{|c|}{ LMW ODCB-2 } & \multicolumn{3}{|c|}{ HMW ODCB-1 } & \multicolumn{3}{|c|}{ HMW ODCB-2 } \\
\hline$v_{1}$ & 1327 & 1327 & 0.06 & 1327 & 1327 & 0.06 & 1327 & 1327 & 0.075 & 1327 & 1327 & 0.06 \\
\hline$v_{2}$ & 1335 & 1335 & 0.13 & 1335 & 1335 & -0.1 & 1335 & 1335 & 0.16 & 1335 & 1335 & -0.10 \\
\hline$v_{3}$ & 1371 & 1371 & 0.26 & 1371 & 1371 & -0.1 & 1371 & 1371 & 0.23 & 1371 & 1371 & -0.10 \\
\hline$v_{4}$ & 1391 & 1391 & 0.30 & 1391 & 1391 & -0.25 & 1391 & 1391 & 0.23 & 1391 & 1391 & -0.10 \\
\hline$v_{5}$ & 1414 & 1414 & 0.36 & 1414 & 1414 & -0.30 & 1414 & 1414 & 0.26 & 1414 & 1414 & -0.10 \\
\hline$v_{6}$ & 1477 & 1477 & 0.28 & 1477 & 1477 & -0.20 & 1477 & 1477 & 0.25 & 1477 & 1477 & -0.15 \\
\hline$v_{7}$ & 1492 & 1492 & 0.56 & 1492 & 1300 & -0.55 & 1492 & 1300 & 0.45 & 1492 & 1300 & -0.50 \\
\hline$v_{8}$ & 1507 & 1507 & 0.21 & 1507 & 1507 & -0.25 & 1507 & 1507 & 0.068 & 1507 & 1507 & 0.068 \\
\hline$v_{9}$ & 1513 & 1513 & 0.38 & 1513 & 1513 & -0.32 & 1513 & 1513 & 0.34 & 1513 & 1513 & -0.38 \\
\hline$\Gamma\left(\mathrm{cm}^{-1}\right)$ & 350 & & & 350 & & & 350 & & & 350 & & \\
\hline$\Theta\left(\mathrm{cm}^{-1}\right)$ & 300 & & & 300 & & & 350 & & & 350 & & \\
\hline$E_{00}\left(\mathrm{~cm}^{1}\right)$ & 12550 & & & 13850 & & & 12400 & & & 13700 & & \\
\hline$M_{l}(\AA)$ & 2.53 & & & 1.74 & & & 2.80 & & & 1.78 & & \\
\hline \multicolumn{4}{|c|}{ LMW CF-1 } & \multicolumn{3}{|c|}{ LMW CF-2 } & \multicolumn{3}{|c|}{ HMW CF-1 } & \multicolumn{3}{|c|}{ HMW CF-2 } \\
\hline$v_{1}$ & 1327 & 1327 & 0.03 & 1327 & 1327 & 0.04 & 1327 & 1327 & 0.06 & 1327 & 1327 & 0.05 \\
\hline$v_{2}$ & 1335 & 1335 & 0.145 & 1335 & 1335 & -0.06 & 1335 & 1335 & 0.165 & 1335 & 1335 & -0.20 \\
\hline$v_{3}$ & 1371 & 1371 & 0.21 & 1371 & 1371 & -0.10 & 1371 & 1371 & 0.255 & 1371 & 1371 & -0.18 \\
\hline$v_{4}$ & 1391 & 1391 & 0.21 & 1391 & 1391 & -0.10 & 1391 & 1391 & 0.23 & 1391 & 1391 & -0.10 \\
\hline$v_{5}$ & 1414 & 1414 & 0.29 & 1414 & 1414 & -0.21 & 1414 & 1414 & 0.27 & 1414 & 1414 & -0.11 \\
\hline$v_{6}$ & 1477 & 1477 & 0.25 & 1477 & 1477 & -0.18 & 1477 & 1477 & 0.25 & 1477 & 1477 & -0.15 \\
\hline$v_{7}$ & 1492 & 1492 & 0.35 & 1492 & 1300 & -0.28 & 1492 & 1300 & 0.42 & 1492 & 1300 & -0.50 \\
\hline$v_{8}$ & 1507 & 1507 & 0.18 & 1507 & 1507 & -0.14 & 1507 & 1507 & 0.07 & 1507 & 1507 & 0.10 \\
\hline$v_{9}$ & 1513 & 1513 & 0.245 & 1513 & 1513 & -0.15 & 1513 & 1513 & 0.35 & 1513 & 1513 & -0.37 \\
\hline$\Gamma\left(\mathrm{cm}^{-1}\right)$ & 300 & & & 300 & & & 350 & & & 350 & & \\
\hline$\Theta\left(\mathrm{cm}^{-1}\right)$ & 450 & & & 450 & & & 350 & & & 350 & & \\
\hline$E_{00}\left(\mathrm{~cm}^{1}\right)$ & 12400 & & & 13700 & & & 12300 & & & 13600 & & \\
\hline$M_{l}(\AA)$ & 2.38 & & & 1.75 & & & 2.79 & & & 1.70 & & \\
\hline
\end{tabular}

\section{CONCLUSION}

Resonance Raman Intensity analysis has provided insight on the effect of polymer molecular weight on the vibronic intensities observed in the absorption spectra of DPP-TT-T. Our analysis indicates that a mixture of conformations, 'trans' and 'cis', is present in solution, with the linear conformation dominating in the HMW polymer driving thus the extinction coefficient to higher values. 


\section{REFERENCES}

[1] Spano, F. C. and Silva, C. "H- and J-Aggregate Behavior in Polymeric Semiconductors," Ann. Rev. Phys. Chem. 65 477-500 (2014).

[2] Vezie, M. S. et al. "Exploring the origin of high optical absorption in conjugated polymers." Nat. Mater. doi:10.1038/nmat4645 (2016).

[3] Myers, A. B. and Mathies, R. A. "Resonance Raman intensities: A probe of excited state structure and dynamics," in Biological Applications of Raman Spectroscopy Vol. 1 (ed T. G. Spiro) (1987).

[4] Bailey, S. E., Cohan, J. S. and Zink, J. I. "Interference effects of multiple excited states in the resonance Raman spectroscopy of CpCoCOD," J. Phys. Chem. B 104, 10743-10749 (2000).

[5] Foster, C. E., Barham, B. P. and Reid, P. J. "Resonance Raman intensity analysis of chlorine dioxide dissolved in chloroform: The role of nonpolar solvation," J. Chem. Phys. 114, 8492-8504 (2001).

[6] Kato, Y. and Takuma, H. "Experimental Study on Wavelength Dependence of Raman Scattering Cross Sections," J. Chem. Phys. 54, 5398-5402 (1971).

[7] Frisch, M. et al. Gaussian 09, revision A. 1; Gaussian, Inc: Wallingford, CT, 2009.

[8] McHale, J. L. Molecular spectroscopy. (Pearson College Division, 1999).

[9] Yan, Y. J. and Mukamel, S. "Eigenstate-free, Green function, calculation of molecular absorption and fluorescence line shapes," J. Chem. Phys. 85, 5908, (1986).

[10] Egolf, D. S., Waterland, M. R. and Myers Kelley, A. "Resonance Raman Intensity Analysis of the Carbazole/Tetracyanoethylene Charge-Transfer Complex: Mode-Specific Reorganization Energies for a HoleTransport Molecule," J. Phys. Chem. B 104, 10727-10737 (2000). 